\title{
Exploring Fear of Cancer Recurrence in a Sample of Heterogeneous Distressed Cancer Patients with and Without a Psychiatric Disorder
}

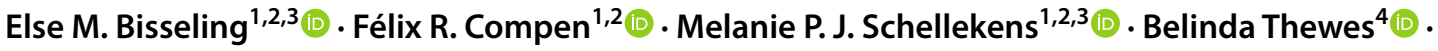 \\ Anne E. M. Speckens ${ }^{1,2}\left(\mathbb{D} \cdot\right.$ Marije L. van der Lee ${ }^{3,5}(1)$
}

Accepted: 2 April 2021 / Published online: 17 June 2021

(c) The Author(s) 2021

\begin{abstract}
Fear of Cancer Recurrence (FCR) is a concern among cancer patients. Recent insights suggest that FCR should be viewed as a distinct syndrome. However, few studies have explored its overlap with psychiatric morbidity. We examined this overlap in a sample of distressed cancer patients. Self-referred patients $(n=245)$ were assessed with the Structured Clinical Interview for DSM-IV-TR Axis-I disorders and the Fear of Cancer Recurrence Inventory-Short Form. Proportions of patients with and without a psychiatric disorder meeting validated cut-offs for screening and clinically relevant FCR were compared. The prevalence of psychiatric disorders was 36\%. Clinically relevant FCR was found in 198 patients (81\%). Patients with a current psychiatric disorder reported clinically relevant FCR more frequently (89\%) compared to those with no disorder (77\%). Of patients reporting clinically relevant FCR, the majority (61\%) did not additionally meet the criteria for a psychiatric disorder. These findings suggest that there should be particular attention for patients with elevated levels of FCR, warranting FCR-specific treatment. Trial registry number Clinicaltrials.gov NCT02138513
\end{abstract}

Keywords Cancer $\cdot$ Oncology $\cdot$ Psychiatric disorder $\cdot$ Psychological distress $\cdot$ Fear of cancer recurrence

\section{Introduction}

Fear of cancer recurrence (FCR) is defined as the "Fear, worry, or concern relating to the possibility that cancer will come back or progress" (Lebel et al., 2016). FCR is one of

Else M. Bisseling

else.bisseling@radboudumc.nl

Belinda Thewes

Belinda@ healthpsychclinic.com.au

1 Department of Psychiatry, Radboud University Medical Centre, 6525, EX, Nijmegen, The Netherlands

2 Department of Psychiatry, Radboud University Medical Centre for Mindfulness, Reinier Postlaan, Postbus 9101, 6500, HB, Nijmegen, The Netherlands

3 Centre for Psycho-Oncology, Scientific Research Department, Helen Dowling Institute, 3723, MB, Bilthoven, The Netherlands

4 School of Psychology, University of Sydney, Sydney, NSW 2006, Australia

5 Department of Medical and Clinical Psychology, School of Social and Behavioral Sciences, Tilburg University, Warandelaan 2, 5037 AB Tilburg, The Netherlands the most prevalent areas of unmet needs in cancer patients (Simard et al., 2013). It has been positively associated with other self-report measures of anxiety symptoms, intrusive thoughts and avoidance and negatively associated with quality of life in a large sample of cancer patients (Simard et al., 2013). A systematic review on FCR in adult cancer patients (Simard et al., 2013) showed that across different cancer sites, 39-97\% of cancer patients reported some degree of FCR, $22-87 \%$ reported a moderate to high degree, and $0-15 \%$ reported severe levels of FCR.

There is debate in the literature whether or not FCR is part of psychiatric disorders and if it should be seen as a distinct syndrome warranting specific intervention. In some countries, like the Netherlands, this debate is becoming significant since evidence-based psychosocial interventions are only reimbursed by health insurers for cancer patients with diagnosable psychiatric disorder.

Thewes and colleagues demonstrated in a sample of 218 young women with breast cancer (Thewes et al., 2013), that most women with screening levels of FCR did not additionally meet screening criteria for generalized anxiety disorder or hypochondriasis. Therefore, the authors suggested that 
FCR should be viewed as a distinct syndrome warranting specific treatment.

However, the relationship between Fear of Cancer Recurrence and established psychiatric disorders is not well understood. Concerns about FCR are among the first symptoms reported by cancer patients who developed a major depression (Kleiboer et al., 2011). Worry and rumination are considered to be the core cognitive processes in anxiety and depressive disorders (Fresco et al., 2002; Nolen-Hoeksema, 2000), which are more prevalent in cancer patients than in the general population (Hartung et al., 2017; Mitchell et al., 2011).Therefore, worry and fear associated with cancer recurrence might be part of known psychiatric disorders such as a major depressive disorder and anxiety disorder.

While several studies have examined the overlap between FCR and psychological symptoms in specific cancer types, only self-report symptom scales or screening instruments were used for assessing psychiatric disorders (MirabeauBeale et al., 2009; Roth et al., 2006; Thewes et al., 2013). Only three studies, with varying results, have examined the relationship between FCR and psychiatric disorders using gold standard psychiatric interview methods. These studies report that $6 \%$ to $40 \%$ of patients with elevated FCR have comorbid psychiatric disorders (Dinkel et al., 2014; Simard \& Savard, 2015; Skaali et al., 2009). However, their findings should be interpreted with caution, as the included studies either measured FCR with one item, FCR was rated with one question measure asking: "During the last week, have you been afraid of relapse of your disease?" (Skaali et al., 2009), used a small sample size $(n=60)$ (Simard \& Savard, 2015) or only anxiety disorders and not depressive disorders were assessed (Dinkel et al., 2014). Furthermore, patients with metastases were excluded from all the above studies. Therefore, generalizability is limited.

The aim of this study is to contribute to understanding of the relationship between Fear of Cancer Recurrence and established psychiatric disorders by (1) comparing the mean level of FCR in cancer patients with and without a psychiatric disorder; and (2) comparing the proportion of patients with and without psychiatric morbidity who meet validated criteria for screening or clinically relevant FCR.

\section{Methods}

\section{Patients and Procedure}

Participants were recruited as part of a three-armed multicenter randomized controlled trial comparing groupbased Mindfulness-Based Cognitive Therapy (MBCT) and individual internet-based MBCT to Treatment As Usual (TAU) (Compen et al., 2018). Inclusion criteria were: (a) a cancer diagnosis, any tumor or stage; $(b) \geq 11$ on the
Hospital Anxiety and Depression Scale (HADS (Bjelland et al., 2002; Spinhoven et al., 1997); (c) computer literacy and internet access; (d) a good command of the Dutch language. Exclusion criteria were: (a) severe psychiatric condition as acute suicidal ideation or psychosis; (b) change in psychotropic medication dosage within a period of three months prior to baseline; and (c) current or previous participation in a mindfulness-based intervention ( $\geq 4$ sessions of MBCT). The study was approved by the local ethics committee and registered on May 6th 2014 (Clinicaltrials.gov NCT02138513). Participants were recruited in participating specialized mental health care institutes for psycho-oncology, via social media, patient associations and advertorials in local newspapers in The Netherlands. Participants who were interested in participation could enroll themselves at the study website at which point they completed the screening assessment (HADS). Participants with HADS $\geq 11$ were contacted by telephone by one of the researchers to assess eligibility. Written informed consent was obtained prior to participation. A subsequent research interview was conducted in which the Structured Clinical Interview for DSM-IV-TR Axis-I disorders (SCID-I; First et al., 2012) was administered to diagnose possible psychiatric disorders. Participants completed remaining (self-report) questionnaires online prior to randomization.

\section{Assessments}

\section{Demographic and Clinical Information}

The following demographic and clinical characteristics were assessed: gender, age, marital status, education, cancer diagnosis, time since cancer diagnosis, current anticancer treatment, current psychopharmacological treatment.

\section{Psychiatric Disorder}

Presence of DSM-IV Axis-I psychiatric disorder was assessed by the Structured Clinical Interview for DSM-IVTR Axis-I disorders (SCID-I; First et al., 2012). The screening module and sections on current and past (recurrent) depressive disorder, current anxiety disorder, and current adjustment disorder were used. The SCID-I was administered by trained interviewers with a master in Behavioral Science (FC) and two Masters-level psychology students. All interviews were audio-taped. Two experienced psychiatrists (EB and AS) and one experienced psychologist (ML) supervised the administration of all the SCID-I interviews and double-rated $(n=98)$ all the audiotapes with positive answers on the 13 item screening module. When there was a discrepancy, the rating was discussed together until consensus was reached. 


\section{Fear of Cancer Recurrence}

Severity of FCR was assessed with the Fear of Cancer Recurrence Inventory-Short Form (FCRI-SF; (Simard \& Savard, 2009a)). The FCRI is a multi-dimensional measure intended for use with cancer patients in all stages and currently one of the strongest measures available. The 9-item severity subscale (total range 1-36), also named FCRI-SF, and has been recommended as a screening tool for high FCR. A cut-off score of $\geq 13$ on the FCRI-SF is associated with the highest sensitivity rates for discovering the presence of significantly high levels of FCR, which enables it to efficiently screen for FCR. A cut-off score of $\geq 16$ on the FCRI-SF is validated as the optimal cut-off point to identify the presence of severe levels of FCR to distinguishing clinically relevant FCR (Simard \& Savard, 2015). In the current study, the Dutch translation of the FCRI was used (FCRISF-NL;(Van Helmondt et al., 2017)). Internal consistency in the present sample was high, Cronbach's alpha $=0.90$.

\section{Differences in Symptoms Measured with FCRI and SCID-I.}

The FCRI was developed by a committee of experts in psycho-oncology and based on a cognitive-behavioral conceptualization of FCR. This instrument assesses how severe the fear of recurrence is, what triggers it, how patients cope with it, how much distress they experience from it, whether it results in functioning impairment, whether patients have insight that they worry too much and how they seek reassurance.

When assessing an adjustment disorder the onset of emotional or behavioral symptoms must occur in response to an identifiable stressor, and within 3 months of the stressor. Distress must be disproportionate to the severity or intensity of the stressor, taking into account contextual and cultural factors and it has to lead to significant impairments in social, occupational or other domains of functioning. The difference with FCR is that FCR can occur outside this timeframe and although the distress related to it may be often understandable and therefore not easily judged to be disproportionate, it may at the same time be so severe that it interferes with functioning. The main difference with depressive disorder is the duration criterion that depressed mood must last at least two weeks and be there most of the day, nearly every day. And the criteria for depressive disorder include a range of somatic symptoms like weight loss, sleep problems, which are not assessed within the FCRI. The main difference with generalized anxiety disorder is again the duration criterion (at least 6 months), and criteria such as being easily fatigued or have difficulty concentrating. The criterion difficulty controlling the worry has overlap with FCR, but in FCR the content of the worry is recurrence, whereas in generalized anxiety disorder, the content of worry is much broader. Thus, the criteria according to DMS are more specific in duration and type of symptoms than the items in the FCRI.

\section{Data Analysis}

Statistical analyses were run in IBM SPSS Statistics version 22. Descriptive statistics were used for the sociodemographic and clinical characteristics, and prevalence rates of current psychiatric disorders. One way ANOVA's with post hoc test were performed to compare mean levels of FCR between groups. A one-way ANOVA was performed with FCR severity as the dependent variable and psychiatric disorder (no versus yes) as fixed effect. A second ANOVA was performed with FCR severity as the dependent variable and type of psychiatric disorder (no/depression disorder/anxiety disorder/adjustment disorder) as fixed effect. Post Hoc Bonferonni tests were conducted to assess whether level of FCR severity differs between the different types of psychiatric disorders. The homogeneity of variance and normality assumption were met.

Proportions of participants with and without a psychiatric disorder meeting validated cut-off for screening and clinically relevant FCR were compared using logistic regression. If a sociodemographic or clinical variable significantly predicted the level of FCR, this variable is added as a control variable in the ANCOVA or logistic regression.

\section{Results}

Of 245 cancer patients eligible for the RCT the majority was female $(85.7 \%)$, diagnosed with breast cancer $(61.6 \%)$ and treated with a curative intent (84.1\%). Mean age of the sample was 51.7 years $(\mathrm{SD}=10.7)$, and mean time since diagnosis was 3.5 years $(\mathrm{SD}=4.7)$. Almost half of participants (47.3\%) had current anticancer treatment; of those, one-third $(32.2 \%)$ had long-term hormonal treatment. See Table 1 for demographic and clinical characteristics.

There were no differences in socio-demographics between participants with or without any psychiatric disorder (see Table 1). However, concerning clinical characteristics, participants with Axis-I psychiatric disorders reported significant higher levels of psychological distress $(M=21.9$, $\mathrm{SD}=5.7)$ compared to those without a psychiatric disorder $(\mathrm{M}=15.2, \mathrm{SD}=5.8(\mathrm{SE}=0.76, p=0.001)$. In addition, they used psychopharmacological medication more often (43.8\%) compared to those without a psychiatric disorder (19.9\%) $\chi^{2}$ $(1, n=245)=15.9, p<0.001$. The mean score on FCRI-SF in the entire sample was $21.3(\mathrm{SD}=6.5)$.

None of the sociodemographic and clinical characteristics significantly predicted FCR levels, except for treatment intent. Participants in palliative care reported significantly higher severity levels $(p=0.002)$ on the FCRI-SF $(\mathrm{M}=24.2$, 
Table 1 Demographic and clinical characteristics of the total sample and divided in absence/presence of psychiatric disorder

\begin{tabular}{|c|c|c|c|c|}
\hline & Total $(n=245)$ & $\begin{array}{l}\text { No psychiatric } \\
\text { disorder }(n=156)\end{array}$ & $\begin{array}{l}\text { Psychiatric disor- } \\
\text { der }(n=89)\end{array}$ & \\
\hline & $M(S D)$ & $M(S D)$ & $M(S D)$ & $P$ \\
\hline Age, $M(S D)$ & $51.7(10.7)$ & $51.8(10.8)$ & $51.4(10.5)$ & .797 \\
\hline Time since diagnosis (years) & $3.5(4.7)$ & $3.4(4.0)$ & $3.6(5.7)$ & .755 \\
\hline Psychological distress level & & & & $<.001$ \\
\hline HADS total & $17.7(6.6)$ & $15.2(5.8)$ & $21.9(5.7)$ & \\
\hline Fear of cancer recurrence & & & & .011 \\
\hline \multirow[t]{2}{*}{ FCRI-SF } & $21.3(6.5)$ & $20.5(6.7)$ & $22.7(5.9)$ & \\
\hline & $N(\%)$ & $N(\%)$ & $N(\%)$ & \\
\hline Gender & & & & .665 \\
\hline Male & $35(14.3)$ & $21(13.5)$ & $14(15.6)$ & \\
\hline Female & $210(85.7)$ & $134(86.5)$ & $76(84.4)$ & \\
\hline Relationship & & & & .143 \\
\hline Yes: Married, living together & $202(82.4)$ & $132(85.2)$ & $70(77.8)$ & \\
\hline No: Divorced / widow/ single & 43(17.6) & $23(14.8)$ & $20(22.2)$ & \\
\hline Educational level & & & & .575 \\
\hline Low/Intermediate & $79(32.2)$ & $48(31.0)$ & $31(34.4)$ & \\
\hline High & $166(67.8)$ & $107(69.0)$ & $59(65.6)$ & \\
\hline Diagnosis & & & & .179 \\
\hline Breast & 151(61.6) & $97(62.2)$ & $54(60.0)$ & \\
\hline Gynecological & $18(7.3)$ & $14(9.0)$ & $4(4.4)$ & \\
\hline Colon & $12(4.9)$ & $9(5.8)$ & $4(3.3)$ & \\
\hline Non-Hodgkin & $11(4.5)$ & $8(5.2)$ & $3(3.3)$ & \\
\hline Prostate & $16(6.5)$ & $10(6.5)$ & $6(6.7)$ & \\
\hline Other & $37(15.1)$ & $17(11.0)$ & $20(22.2)$ & \\
\hline Treatment intent & & & & .399 \\
\hline Curative & $206(84.1)$ & $128(82.6)$ & $78(86.7)$ & \\
\hline Palliative & 39 (15.9) & $27(17.4)$ & $12(13.3)$ & \\
\hline Active anticancer treatment & & & & .962 \\
\hline None & $129(52.7)$ & $81(52.3)$ & $48(53.3)$ & \\
\hline Hormone & $79(32.2)$ & $48(31.0)$ & $31(34.4)$ & \\
\hline Chemotherapy/Radiation & $12(4.9)$ & $8(4.1)$ & $4(4.4)$ & \\
\hline Other & $23(10.4)$ & $18(1.6)$ & $7(7.7)$ & \\
\hline Psychopharmacological treatment & & & & $<.001$ \\
\hline Yes & $70(28.6)$ & 31(19.9) & $39(43.8)$ & \\
\hline No & $175(71.4)$ & $125(80.1)$ & $50(56.2)$ & \\
\hline
\end{tabular}

$\mathrm{SD}=6.4)$, than those who were treated with curative intent $(\mathrm{M}=20.7, \mathrm{SD}=6.4)$.

\section{Psychiatric Morbidity}

Of the SCID-I interviews with the 245 participants, 89 $(36 \%)$ participants met the criteria for a current psychiatric disorder. Of those, 42 (47\%) met the criteria of a depressive disorder, 27 (30\%) had an anxiety disorder, 20 (23\%) had an adjustment disorder. Five (6\%) participants had concurrent depressive and anxiety disorders (see Table 2).
Table 2 Prevalence of psychiatric disorders and mean level of FCR

\begin{tabular}{llll}
\hline & $\mathrm{N}$ & $\%(95 \% \mathrm{CI})$ & $\mathrm{M}(\mathrm{SD}) \mathrm{FCR}$ \\
\hline No disorder & 156 & & 20.5 \\
Any disorder & 89 & 100 & $22.7(5.9)$ \\
-Depressive & 42 & $47.2(37-57)$ & $22.3(6.1)$ \\
-Anxiety & 27 & $30.3(22-41)$ & $23.3(4.8)$ \\
-Adjustment & 20 & $22.5(15-32)$ & $22.8(6.8)$ \\
\hline
\end{tabular}




\section{Mean Levels of FCR in Cancer Patients with and Without Psychiatric Diagnosis}

On average, participants with a psychiatric disorder reported significantly higher severity levels $(p=0.011)$ on the FCRI$\mathrm{SF}(\mathrm{M}=22.7, \mathrm{SD}=0.62)$, than those without a current psychiatric disorder $(M=20.5, S D=6.7)$ (see Table 3$)$. Post hoc Bonferonni tests revealed that mean levels of FCR did not significantly differ between specific groups of psychiatric disorders (no disorder, depressive disorder, anxiety disorder, adjustment disorder), $\mathrm{F}=2.318, p=0.076, \eta 2=0.025$. Adding treatment intent (curative versus palliative) as a control variable in the ANCOVA's resulted in similar findings.

\section{Screening Level (High) FCR and Clinically Relevant (Severe) FCR in Cancer Patients with and Without Psychiatric Diagnosis}

Two hundred and twenty-one participants (91\%) scored above the FCRI-SF screening cut-off ( $\geq 13$ ) (see Table 4). Almost all participants (97\%) with a current Axis-I disorder scored in the high range $(\geq 13)$ for FCR. Thus participants with any Axis-I disorder were significantly more likely to score above FCRI-SF screening cut-off $(\geq 13)$ than participants without an Axis-I disorder, $(\mathrm{OR}=4.17, p=0.024)$. Adding treatment intent (curative versus palliative) as a control variable in the logistic regression analysis resulted in similar findings.

Regarding this level of high FCR, the majority of participants (61\%) did not additionally meet the criteria for a psychiatric disorder. As almost all of the participants scored above the screening cut-off, the proportion of clinically relevant (severe) FCR was also examined in this sample. Clinically relevant (severe) FCR $(\geq 16)$ was found in 199 participants (81\%). Most participants $(89 \%)$ with a current Axis-I disorder scored in the clinically relevant range $(\geq 16)$ for FCR. Participants with any Axis-I disorder were significantly more likely to score above the threshold for clinically relevant FCR $(\geq 16)$ than participants without an Axis-I disorder, $\mathrm{OR}=2.34, p=0.028$. Adding treatment intent (curative versus palliative) as a control variable in the ANCOVA's resulted in similar findings. Regarding this level of severe FCR, the majority of participants (62\%) did not additionally meet the criteria for a psychiatric disorder.

\section{Discussion}

The aim of our study was to better understand the relationship between FCR and psychiatric morbidity by describing the prevalence and nature of psychiatric morbidity, comparing the mean level of FCR in heterogeneous cancer patients with and without psychiatric diagnosis and exploring the overlap of high and severe FCR with psychiatric morbidity.

We found significant differences in mean levels of FCR between participants, such that having a psychiatric disorder or being in a palliative stage was associated with elevated mean levels of FCR. Similar to previous studies in curative treated cancer patients, the prevalence of psychiatric disorders in this sample was approximately $40 \%$ (Kuhnt et al., 2016; Mitchell et al., 2011). Cancer patients in palliative care were found to be at greater risk for developing elevated
Table 3 Fixed effects ANOVA results with FCRI-SF as dependent variable

\begin{tabular}{llllrll}
\hline Predictor & Sum of squares & Df & Mean square & F & $P$ & $\eta^{2}$ \\
\hline Intercept & $104,786,03$ & 1 & $104,786,03$ & 2531.77 & .000 & \\
Psychiatric Disorder & 274,16 & 1 & 274,16 & 6.62 & .011 & .025 \\
Error & $10,015,99$ & 242 & 41,39 & & & \\
\hline
\end{tabular}

Table 4 Screening level (high) FCR and clinically relevant (severe) FCR in cancer patients with and without psychiatric diagnosis

\begin{tabular}{|c|c|c|c|c|c|}
\hline & \multirow{2}{*}{$\begin{array}{l}\text { No disorder } \\
(n=156) n(\%)\end{array}$} & \multicolumn{4}{|l|}{ Disorder } \\
\hline & & $\begin{array}{l}\text { Any Disorder } \\
(n=88)^{\mathrm{a}} n(\%)\end{array}$ & $\begin{array}{l}\text { Depressive } \\
(n=42) n(\%)\end{array}$ & $\begin{array}{l}\text { Anxiety } \\
(n=26)^{\mathrm{a}} n \\
(\%)\end{array}$ & $\begin{array}{l}\text { Adjustment } \\
(n=20) n \\
(\%)\end{array}$ \\
\hline \multicolumn{6}{|l|}{ Screening level } \\
\hline$\%<13$ FCR $(n=23)$ & $20(12.8)$ & $3(3.4)$ & $2(4.8)$ & $0(0.0)$ & $1(5.0)$ \\
\hline$\% \geq 13$ FCR $(n=221)^{\mathrm{b}}$ & $136(87.2)$ & $85(96.6)$ & $40(95.2)$ & $26(100.0)$ & $19(95.0)$ \\
\hline \multicolumn{6}{|l|}{ Clinically relevant } \\
\hline$\%<16$ FCR $(n=46)$ & $36(23.1)$ & $10(11.4)$ & $5(11.9)$ & $2(7.7)$ & $3(15.0)$ \\
\hline$\% \geq 16$ FCR $(n=198)^{\mathrm{b}}$ & $120(76.9)$ & $78(88.6)$ & $37(88.1)$ & $24(92.3)$ & $17(85.0)$ \\
\hline
\end{tabular}

${ }^{\mathrm{a}} n=26$ rather than 27 (see Table2) because one person with an anxiety disorder did not fill out the FCRI.

${ }^{\mathrm{b}}$ This cumulates to 244 because one participant did not fill out the FCRI 
levels of FCR, but a palliative stage of disease was not associated with increased likelihood of psychiatric disorder. Over $80 \%$ of the sample showed clinically relevant FCR. This is higher than has been reported in a review on FCR in general cancer populations (49\%) (Simard et al., 2013) and higher than previously found in a population of breast cancer patients (70\%) (Thewes et al., 2012) and could be explained by the fact that this sample consisted of help-seeking distressed cancer patients.

Our findings confirm earlier research findings that FCR is a common distressing phenomenon in cancer patients (Fardell et al., 2016, 2017; Simard et al., 2013), and that it is highly prevalent in patients with psychiatric morbidity (Mitchell et al., 2011; Simard \& Savard, 2009b, 2015; Thewes et al., 2013) and patients in palliative care (Wal et al., 2016). While the vast majority of patients with psychiatric disorder experience elevated levels of FCR, the majority of patients with elevated FCR do not experience psychiatric disorder. We replicated Simard's findings (Simard \& Savard, 2015) that high FCR is comorbid with psychiatric morbidity over one-third of cases. It was found that high FCR is comorbid with depressive disorders as well as anxiety disorders. It could be that having high levels of FCR might be a prodromal or atypical symptom of a range of Axis-I psychiatric disorders in cancer patients. Therefore, longitudinal studies are needed to disentangle the causal and temporal relationship between FCR and psychiatric morbidity.

As clinically relevant FCR was found in $80 \%$ of cases in the present sample, a higher cut-off, even up to $\geq 22$ (Fardell et al., 2017), might be needed to clearly distinguish cancer patients in need of more intensive FCR-specific interventions.

\section{Study Strengths and Limitations}

A major strength of this study was the use of the gold standard to assess psychiatric disorders. Two experienced psychiatrists and one experienced psychologist supervised and double-rated the SCID-I interviews and particular emphasis was placed on detecting psychiatric disorders. Another strength was that this research was conducted in a relative large sample of heterogeneous cancer patients with respect to cancer type and prognosis. Despite these strengths there are some noteworthy limitations. The present study was conducted in a group of distressed help-seeking cancer patients. Although this patient-centered nature of recruitment resulting in a convenience sample might benefit generalization of research findings to clinical practice, the self-selection inherent in this sampling method threatens internal validity and can be seen as a drawback, limiting the extent to which the present findings generalize to the broader population of cancer patients. We did not assess past anxiety disorders while it could be that high FCR is another manifestation of for example Generalized Anxiety Disorder, but with the anxiety focused on cancer recurrence. Future research should assess past anxiety disorders to know whether high FCR is a clinical manifestation of pre-existing anxiety disorder.

\section{Clinical Implications}

Fear and anxiety are part of cancer survivorship. Specific worry about cancer recurrence is one of the most common concerns of cancer patients. We found that the majority of cancer patients with high FCR do not have psychiatric morbidity, suggesting that FCR is a distinct syndrome, worthy of specific interventions. It is to be discussed whether high FCR warrants a separate DSM category, or that it fits in within existing categories like illness anxiety. We recommend that future studies will also look at the criterion of significant impairments in social, occupational, or other domains of functioning specifically. Patients with elevated levels of FCR might benefit from a stepped care approach, involving strategies such as watchful waiting or psycho-education for low to moderate levels of FCR, self-management interventions for moderate to high levels of FCR, and evidence-based faceto-face psychological interventions (Butow et al., 2017; Herschbach et al., 2010; Smith et al., 2015) or blended therapy approaches (van de Wal, et al., 2017) for patients with severe FCR. Therefore, greater accessibility of FCR-specific treatment programs is a priority. In some countries, evidencebased psychosocial interventions are only reimbursed by health insurers for cancer patients with diagnosable psychiatric disorder. The findings of the present study have specific relevance for the way in which FCR interventions are funded and how they are implemented. The present study found that over one-third of cases of severe FCR overlaps with Axis-I psychiatric morbidity. Therefore, whenever necessary, referral to a clinical psychologist or psychiatrist should be offered when severe FCR levels are identified.

Acknowledgements This research is funded by a grant from Pink Ribbon (2012.WO14.C153) awarded to Prof. Dr. Anne E.M. Speckens and Prof. Dr. Marije L. van der Lee. We are grateful to the patients for their participation in this trial and to physicians and therapists from Radboud University Medical Centre, Nijmegen; Helen Dowling Institute, Bilthoven; De Vruchtenburg, Leiden/Rotterdam; Ingeborg Douwes Centrum, Amsterdam; Het Behouden Huys, Haren; and Jeroen Bosch Ziekenhuis, Den Bosch for referring patients to the trial. We would like to thank Ms. Heidi Willemse, Eva Witteveen, Merel Brands, and Mr. David Huijts for their help in data collection.

\section{Declarations}

Conflict of interest Authors Else M. Bisseling, Félix R. Compen, Melanie P.J. Schellekens, Belinda Thewes, Anne E.M. Speckens, and Marije L. van der Lee declare that they have no conflict of interest. 
Ethical approval The study was approved by the ethical review board of the Radboud University Medical Center (CMO ArnhemNijmegen 2013/542). All procedures performed in studies involving human participants were in accordance with the ethical standards of the institutional and/or national research committee and with the 1964 Helsinki declaration and its later amendments or comparable ethical standards.

Informed consent Informed consent was obtained from all individual participants included in the study.

Open Access This article is licensed under a Creative Commons Attribution 4.0 International License, which permits use, sharing, adaptation, distribution and reproduction in any medium or format, as long as you give appropriate credit to the original author(s) and the source, provide a link to the Creative Commons licence, and indicate if changes were made. The images or other third party material in this article are included in the article's Creative Commons licence, unless indicated otherwise in a credit line to the material. If material is not included in the article's Creative Commons licence and your intended use is not permitted by statutory regulation or exceeds the permitted use, you will need to obtain permission directly from the copyright holder. To view a copy of this licence, visit http://creativecommons.org/licenses/by/4.0/.

\section{References}

Bjelland, I., Dahl, A. A., Haug, T. T., \& Neckelmann, D. (2002). The validity of the hospital anxiety and depression scale. An updated literature review. Journal of Psychosom Research, 52(2), 69-77

Butow, P. N., Turner, J., Gilchrist, J., Sharpe, L., Smith, A. B., Fardell, J. E., \& Thewes, B. (2017). Randomized trial of conquerfear: A novel, theoretically based psychosocial intervention for fear of cancer recurrence. Journal of Clinical Oncology, 35(36), 4066-4077

Compen, F., Bisseling, E., Schellekens, M., Donders, R., Carlson, L., van der Lee, M., \& Speckens, A. (2018). Face-to-face and internet-based mindfulness-based cognitive therapy compared with treatment as usual in reducing psychological distress in patients with cancer: A multicenter randomized controlled trial. Journal of Clinical Oncology, 36(23), 2413-2421

Dinkel, A., Kremsreiter, K., Marten-Mittag, B., \& Lahmann, C. (2014). Comorbidity of fear of progression and anxiety disorders in cancer patients. General Hospital Psychiatry, 36(6), 613-619

Fardell, J. E., Jones, G., Smith, A. B., Lebel, S., Thewes, B., Costa, D., \& Butow, P. (2017). Exploring the screening capacity of the fear of cancer recurrence inventory-short form for clinical levels of fear of cancer recurrence. Psycho-Oncology. https://doi.org/ 10.1002/pon.4516

Fardell, J. E., Thewes, B., Turner, J., Gilchrist, J., Sharpe, L., Smith, A., \& Butow, P. (2016). Fear of cancer recurrence: a theoretical review and novel cognitive processing formulation. Journal of Cancer Survivorship, 10(4), 663-673. https://doi.org/10.1007/ s11764-015-0512-5

First, M. B., Spitzer, R. L., Gibbon, M., \& Williams, J. B. (2012). Structured clinical interview for DSM-IV® axis i disorders (SCID-I). Administration booklet. American Psychiatric Pub: Clinician Version.

Fresco, D. M., Frankel, A. N., Mennin, D. S., Turk, C. L., \& Heimberg, R. G. (2002). Distinct and overlapping features of rumination and worry: The relationship of cognitive production to negative affective states. Cognitive Therapy and Research, 26(2), 179-188. https://doi.org/10.1023/a:1014517718949
Hartung, T. J., Brahler, E., Faller, H., Harter, M., Hinz, A., Johansen, C., \& Mehnert, A. (2017). The risk of being depressed is significantly higher in cancer patients than in the general population: Prevalence and severity of depressive symptoms across major cancer types. European Journal of Cancer, 72, 46-53. https:// doi.org/10.1016/j.ejca.2016.11.017

Herschbach, P., Book, K., Dinkel, A., Berg, P., Waadt, S., Duran, G., \& Henrich, G. (2010). Evaluation of two group therapies to reduce fear of progression in cancer patients. Supportive Care in Cancer, 18(4), 471-479. https://doi.org/10.1007/s00520-009-0696-1

Kleiboer, A., Bennett, F., Hodges, L., Walker, J., Thekkumpurath, P., $\&$ Sharpe, M. (2011). The problems reported by cancer patients with major depression. Psycho-Oncology, 20(1), 62-68. https:// doi.org/10.1002/pon.1708

Kuhnt, S., Brahler, E., Faller, H., Harter, M., Keller, M., Schulz, H., $\&$ Mehnert, A. (2016). Twelve-month and lifetime prevalence of mental disorders in cancer patients. Psychotherapy and Psychosomatics, 85(5), 289-296. https://doi.org/10.1159/000446991

Lebel, S., Ozakinci, G., Humphris, G., Mutsaers, B., Thewes, B., Prins, J., \& Butow, P. (2016). From normal response to clinical problem: Definition and clinical features of fear of cancer recurrence. Supportive Care in Cancer, 24(8), 3265-3268. https://doi.org/10. 1007/s00520-016-3272-5

Mirabeau-Beale, K. L., Kornblith, A. B., Penson, R. T., Lee, H., Goodman, A., Campos, S. M., \& Matulonis, U. A. (2009). Comparison of the quality of life of early and advanced stage ovarian cancer survivors. Gynecologic Oncology, 114(2), 353-359. https://doi. org/10.1016/j.ygyno.2009.05.009

Mitchell, A. J., Chan, M., Bhatti, H., Halton, M., Grassi, L., \& Johansen, C. (2011). Prevalence of depression, anxiety, and adjustment disorder in oncological, haematological, and palliative-care settings: A meta-analysis of 94 interview-based studies. The Lancet Oncology, 12, 160-174

Nolen-Hoeksema, S. (2000). The role of rumination in depressive disorders and mixed anxiety/depressive symptoms. Journal of Abnormal Psychology, 109(3), 504-511

Roth, A., Nelson, C. J., Rosenfeld, B., Warshowski, A., O’Shea, N., Scher, H., \& Breitbart, W. (2006). Assessing anxiety in men with prostate cancer: further data on the reliability and validity of the Memorial Anxiety Scale for Prostate Cancer (MAX-PC). Psychosomatics, 47(4), 340-347. https://doi.org/10.1176/appi.psy. 47.4.340

Simard, S., \& Savard, J. (2009a). Fear of Cancer Recurrence Inventory: development and initial validation of a multidimensional measure of fear of cancer recurrence. Supportive Care in Cancer, 17(3), 241-251. https://doi.org/10.1007/s00520-008-0444-y

Simard, S., \& Savard, J. (2009b). Fear of cancer recurrence inventory: Development and initial validation of a multidimensional measure of fear of cancer recurrence. Supportive Care in Cancer, $17,241-251$

Simard, S., \& Savard, J. (2015). Screening and comorbidity of clinical levels of fear of cancer recurrence. Journal of Cancer Survivorship, 9(3), 481-491. https://doi.org/10.1007/s11764-015-0424-4

Simard, S., Thewes, B., Humphris, G., Dixon, M., Hayden, C., Mireskandari, S., \& Ozakinci, G. (2013). Fear of cancer recurrence in adult cancer survivors: A systematic review of quantitative studies. Journal of Cancer Survivorship, 7(3), 300-322. https://doi. org/10.1007/s11764-013-0272-z

Skaali, T., Fossa, S. D., Bremnes, R., Dahl, O., Haaland, C. F., Hauge, E. R., \& Dahl, A. A. (2009). Fear of recurrence in long-term testicular cancer survivors. Psycho-Oncology, 18(6), 580-588. https://doi.org/10.1002/pon.1437

Smith, A., Thewes, B., Turner, J., Gilchrist, J., Fardell, J., Sharpe, L., \& Butow, P. (2015). Pilot of a theoretically grounded psychologist-delivered intervention for fear of cancer recurrence (Conquer 
Fear). Psycho-Oncology, 24(8), 967-970. https://doi.org/10.1002/ pon. 3775

Spinhoven, P., Ormel, J., Sloekers, P. P. A., Kempen, G. J. M., Speckens, A. E. M., \& Van Hemert, A. M. (1997). A validation study of the Hospital Anziety and Depression Scale (HADS) in different groups of Dutch subject. Psychological Medicine, 27, 363-370

Thewes, B., Bell, M. L., Butow, P., Beith, J., Boyle, F., Friedlander, M., \& McLachlan, S. A. (2013). Psychological morbidity and stress but not social factors influence level of fear of cancer recurrence in young women with early breast cancer: Results of a cross-sectional study. Psycho-Oncology, 22(12), 2797-2806. https://doi. org/10.1002/pon.3348

Thewes, B., Butow, P., Bell, M. L., Beith, J., Stuart-Harris, R., Grossi, M., \& Dalley, D. (2012). Fear of cancer recurrence in young women with a history of early-stage breast cancer: A cross-sectional study of prevalence and association with health behaviours. Supportive Care in Cancer, 20(11), 2651-2659. https://doi.org/ 10.1007/s00520-011-1371-x van de Wal, M., Thewes, B., Gielissen, M., Speckens, A., \& Prins, J. (2017). Efficacy of blended cognitive behavior therapy for high fear of recurrence in breast, prostate, and colorectal cancer survivors: The SWORD study, a randomized controlled trial. Journal of Clinical Oncology, 35(19), 2173-2183. https://doi.org/10.1200/ jco.2016.70.5301

van Helmondt, S. J., van der Lee, M. L., \& de Vries, J. (2017). Translation and validation of the Dutch version of the Fear of Cancer Recurrence Inventory (FCRI-NL). Journal of Psychosomatic Research. https://doi.org/10.1016/j.jpsychores.2017.09.001

Wal, M., Poll-Franse, L., Prins, J., \& Gielissen, M. (2016). Does fear of cancer recurrence differ between cancer types? A study from the population-based PROFILES registry. Psycho-Oncology, 25(7), $772-778$

Publisher's Note Springer Nature remains neutral with regard to jurisdictional claims in published maps and institutional affiliations. 